\title{
Outcome of Head Injury Patients Undergoing Surgical Management: A Tertiary Level Experience
}

\author{
Shrestha A, Joshi R M, Thapa A, Devkota UP, Gongal DN
}

National Institute of Neurological and Allied Sciences

Bansbari, Kathmandu.

Corresponding author:

Ajit Shrestha

National Institute of Neurological and Allied Sciences

Bansbari, Kathmandu

email: ajitligal@gmail.com

\section{Citation}

Shrestha A, Joshi R M, Thapa A, Devkota UP, Gongal DN. Outcome of Traumatic Brain Injury in Head Injury PatientsUndergoing Surgical Management: A Tertiary Level Neuro-centre Experience. Kathmandu Univ Med J 2011;3364):283-5.

\author{
ABSTRACT \\ Background \\ Head injury is the major cause of death in a neurosurgical patient.
}

\section{Objective}

To find the outcome, and treatment modality affecting the outcome in patients with head injury.

\section{Methods}

Nine hundred eighty seven patients presenting to National Institute of Neurological and Allied Sciences, Kathmandu, with head injury from September 2009 to October 2010 were included in the study. Patients were categorized according to post resuscitation Glasgow Coma Score. Outcome was assessed at discharge using Glasgow Outcome Score and analyzed for any correlation with modality of treatment and severity of injury.

\section{Results}

Among 987 patients with head injury,152 (15.4\%) had severe, $126(12.8 \%)$ had moderate and $709(71.8 \%)$ had mild head injuries. Three hundred twelve (31.6\%) patients required definitive and supportive surgical intervention. One hundred eighty two required cranial surgical intervention. Overall mortality was 10\% (99), 137 patients (13.9\%) had unfavorable outcome and 850 (86.1\%) had favorable Glasgow Outcome Score of 4 and 5 . Mortality was 53.2\%, 9.5\% and $0.8 \%$ in severe, moderate and mild head injury group respectively. Mortality rate was significantly higher $(64.6 \%)$ in severe head injury group managed conservatively than those in same group treated with supportive and definite surgical intervention (44.8\%) $(p=0.016)$.

\section{Conclusion}

Mortality in head injury patients depend upon severity of injury. Mortality in severe head injury group can be reduced by supportive and definite surgical intervention.

\section{KEY WORDS}

Glasgow Outcome, Head injury, Surgical intervention

\section{INTRODUCTION}

Head injury is the commonest cause of death and disability following injury worldwide. It is the cause of death in more than $50 \%$ of trauma patients as discussed by Castillo M. ${ }^{1}$ This holds true in our context too. Fall and road traffic accidents are major causes of head injuries in Nepal. ${ }^{2}$ Usually less severe head injuries are managed at primary level or referring hospitals and only severe head injuries or those requiring surgical interventions are referred to tertiary level neurocentre.
The outcome of head injury depends upon severity of injury amongst other factors. Appropriate and timely interventions in severe injuries can help reduce the mortality of head injuries. This study is aimed at evaluating the outcome of head injury depending upon the post resuscitation Glasgow Coma Scale of patient and role of definite and supportive surgical intervention in severe head injuries. 


\section{METHODS}

Non-probablility purposive sampling technique was followed and a prospective longitudinal analytical study was performed. A structured proforma for data collection was prepared and data of study cohort was entered in the proforma. This study was performed as per the guidelines laid by the institutional review board of NINAS and ethical approval was obtained from the board.

All patients attending to emergency department of National Institute of Neurological and Allied Sciences, Bansbari, Kathmandu with a history of head injury was enrolled in the study. Patients with clinical evidence of brain death at the time of arrival and who didn't revive after resuscitation in the emergency were excluded from the study.

A total of 987 patients were included in the study from September 2009 to October 2010. Patients were resuscitated in the hospital and post resuscitation Glasgow Coma Score was recorded. Patients were then categorized into mild head injury (GCS 13-15), moderate head injury (GCS 9-12) and severe head injury (GCS 3-8) and managed as per the institutional protocol. Surgical intervention was provided as definite or supportive interventions according to the patient's requirement. Outcome was assessed at discharge using Glasgow Outcome Score and analyzed for any correlation with modality of treatment and severity of injury. Statistical analysis was done using SPSS version 16 taking $\mathrm{p}$-value $<0.05$ as significant.

\section{RESULTS}

Total 2921 patients attended the emergency department of National Institute of Neurological and Allied Sciences, Bansbari, Kathmandu during the study period among whom 987 (33.8\%) had head injury. Out of 987 patients with head injuries, $711(72 \%)$ patients were admitted to the hospital.

Age group of patients was in the range of 1 month to 95 years with mean age of $28.5+18.96$ years. Most commonly affected age group was in the range of $20-30$ years (24.5\%). $784(75.8 \%)$ of patients were male. Road traffic accidents was the cause of injury in 541 (54.8\%) patients, followed by fall from height in 299 (30.3\%), physical assault in 125 $(12.7 \%)$ and other cause in rest 22 (2.2\%).

Patients were categorized into three groups according to the post resuscitation GCS. 709 (71.8\%) patients had minor head injury, $126(12.8 \%)$ had moderate head injury and 152 (15.4\%) had severe head injury. 278 (28.2\%) patients had polytrauma and 31 (3.1\%) patients has associated spinal injury. Hospital stay of the patients ranged from 1-120 days with mean duration of $7+10.47$ days.

Surgical intervention was performed in 312 (31.6\%) patients out of whom 182 required cranial surgical intervention. Thirty one (3.1\%) patients had surgeries for elevation of depressed fracture, 104 (10.5\%) had craniotomy for removal of hematoma and contusions, 29
(2.9\%) had burr hole and ICP monitoring and 18 (1.8\%) had decompressive craniectomy. 201 patients, including some patients who had definite surgery, had supportive surgeries like tracheostomy, feeding gastrostomy and surgeries for polytrauma. Among severe head injury patients, 87 (57.2\%) patients required some form of surgical intervention as shown in Table 1.

Table 1. Surgical interventions in different head injury groups.

\begin{tabular}{llll} 
Severity of Head Injury & \multicolumn{2}{l}{ Surgical Intervention } & Total \\
& Present (\%) & None (\%) & \\
\hline Severe & $87(57.2)$ & $65(42.8)$ & 152 \\
\hline Moderate & $54(42.8)$ & $72(57.2)$ & 126 \\
\hline Mild & $171(24.1)$ & $538(75.9)$ & 709 \\
\hline Total & 312 & 675 & 987
\end{tabular}

Overall mortality in the study cohort was $10 \%$ (99 cases). 137 patients $(13.9 \%)$ had unfavorable outcome (GOS 1-3) and 850 (86.1\%) had favorable outcome (GOS 4-5) as shown in Table 2.

Table 2. Glasgow Outcome Score in study cohort.

\begin{tabular}{ll}
\hline Glasgow Outcome Score & Number (\%) \\
\hline 1. Death & $99(10)$ \\
\hline 2. Vegetative & $12(1.2)$ \\
\hline 3. Severe Disability & $26(2.6)$ \\
\hline 4. Moderate Disability & $158(16.1)$ \\
\hline 5. Good Recovery & $692(70.1)$ \\
\hline Total & 987
\end{tabular}

Mortality was $53.2 \%, 9.5 \%$ and $0.8 \%$ in severe, moderate and mild head injury group respectively. Mortality rate was significantly higher (64.6\%) in severe head injury group managed conservatively than in severe head injury patients treated with supportive and definite surgical intervention (44.8\%) $(p=0.016)$ as shown in Table 3.

Table 3. Outcome in different head injury groups in relation to surgical interventions.

\begin{tabular}{|llllll|}
$\begin{array}{l}\text { Severity of } \\
\text { Head Injury }\end{array}$ & $\begin{array}{l}\text { Surgical Inter- } \\
\text { ventions }\end{array}$ & Outcome & Total & $\begin{array}{l}\mathbf{p} \\
\text { value }\end{array}$ \\
\hline $\begin{array}{l}\text { Severe } \\
\text { Head Injury }\end{array}$ & None & 42 & 23 & 65 & 0.016 \\
& Present & 39 & 48 & 87 & \\
& Total & 81 & 71 & 152 & \\
\hline Moderate & None & 7 & 65 & 72 & 0.591 \\
\hline Head Injury & Present & 5 & 49 & 54 & \\
& Total & 12 & 114 & 126 & \\
\hline Mild Head & None & 3 & 535 & 538 & 0.137 \\
\hline Injury & Present & 3 & 168 & 171 & \\
& Total & 6 & 703 & 709 & \\
\hline
\end{tabular}




\section{DISCUSSION}

Outcome of head injury correlates with severity of injury as has been shown by different literatures published till date. ${ }^{3-}$ ${ }^{8}$ We used Glasgow Coma Scale and Glasgow Outcome Score to analyze the outcome of head injury in our study too.

Mortality in severe head injury patients vary considerably. Jennett et al published that mortality in severe head injury was $49 \%$ where as Fakhrey et al found it to be $28.8 \% .^{3,6}$ Our study has overall mortality of $10 \%$ and mortality for severe head injury group of $53.2 \%$. Mortality, however, varies according to GCS score even in the severe head injury group. In present study, 43 patients had GCS of 3-4 at the time of presentation out of which 32 (74.4\%) died within 12 hour of presentation. Marshall et a' has shown in his study that $78 \%$ of severe head injury patients with GCS of 3-4 died. $^{7}$

In patients with severe head injury, surgical intervention had a statistically better outcome compared to those who were not operated. $64.6 \%$ of patients with severe head injury managed conservatively died whereas only $44.8 \%$ with surgical intervention succumbed $(p=0.016)$. Similar findings were published by Ahmed $\mathrm{S}$ et al where $72.2 \%$ of patient with severe head injury, who were managed conservatively, died, compared to $23.7 \%$ in patients group managed surgically, though they have not mentioned what kind of intervention were performed in the operated group of patients. ${ }^{7}$ Our study shows that surgical intervention in severe head injury group results in better outcome compared to conservative management. Similar to previous studies, this study still lack some definite evidence of role of supportive surgery only over definitive surgery to treat primary and secondary brain injuries in such patients. A limitation of our study is that some of the patients with GCS 3 and dilated, non reacting pupils for more than six hours were treated conservatively without any kind of surgical intervention.

\section{CONCLUSION}

Outcome in head injury depends upon post resuscitation GCS of the patient. Severe head injury patients have highest mortality rate, however, our findings suggest that mortality in severe head injury group can be reduced by appropriate surgical intervention including definite surgery to treat the primary or secondary brain injury or supportive surgery like tracheostomy, feeding gastrostomy and others. It is felt that larger prospective study including type of surgical intervention to identify relation with supportive surgery in such patients would be more useful.

\section{REFERENCES}

1. Castillo $M$, Harris JH. Skull and brain. In: Harris JH, Harris $\mathbf{W H}$, Novelline AR, editors. The radiology of emergency medicine. Baltimore: Williams and Wilkins; 1993.

2. McClennan S, Snider C. Head injuries in Kathmandu, Nepal. McMaster Univ Med J 2003; 1: 10-14.

3. Jennett Brian, Teasdale G, Braakman R, et al. Predicting outcome in individual patients after severe head injury. Lancet 1976;1:1031-4.

4. Gomez PA, Lobato RD, Boto GR et al. Age and outcome after severe head injury. Acta Neurochir 2000;142: 373-81.
5. Murray LS, Teasdale GM, Murray GD et al. Does prediction of outcome alter patient management? Lancet 1993;34:1487-91.

6. Fakhry SM, Trask AL, Waller MA, Watts DD. Management of braininjured patients by an evidence-based medicine protocol improves outcomes and decreases hospital charges. J Trauma 2004;56: 492-9.

7. Marshall LF, Gautille T, Klaube P, et al. The outcome of severe closed head injury. J Neurosurg 1991;75(5):S28-S36.

8. Ahmed S, Khan S, Agrawal D, Sharma BS. Out come in Head Injured patients: Experience at a level 1 Trauma Centre. In J Neurotrauma 2009;6(2):119-22. 